\title{
Mutant prevention concentration of tigecycline for Klebsiella pneumoniae isolates with four different resistance statuses
}

\author{
Xue Zhong ${ }^{1,2}$, Hong-Tao Xü ${ }^{3}$, Dong-Ke Chen ${ }^{3}$, Xin $\mathrm{Hu}^{2}$, Gang Cheng ${ }^{1}$ \\ ${ }^{1}$ Department of Clinical Pharmacy, School of Pharmacy, Shenyang Pharmaceutical University, Shenyang Liaoning, \\ China \\ ${ }^{2}$ Department of Pharmacy, Beijing Hospital, Beijing, China \\ ${ }^{3}$ Department of Clinical Laboratory, Beijing Hospital, Beijing, China
}

Key words: tigecycline; Klebsiella pneumoniae; mutant prevention concentration.

J Infect Dev Ctries 2015; 9(10):1177-1179. doi:10.3855/jidc.7156

(Received 18 May 2015 - Accepted 09 July 2015)

Copyright (C) 2015 Zhong et al. This is an open-access article distributed under the Creative Commons Attribution License, which permits unrestricted use, distribution, and reproduction in any medium, provided the original work is properly cited.

Dear Editor,

Klebsiella pneumoniae (K. pneumoniae) is one of the most important causes of healthcare-associated infections, especially among hospitalized patients [1]. Since most clinical isolates of $K$. pneumoniae are resistant to fluoroquinolones and carbapenems, clinicians have to use tigecycline as the last resort. Unfortunately, the prevalence of tigecycline-resistant $K$. pneumoniae seems to be increasing [2,3]. The potential development of resistance to tigecycline during treatment is of concern. In this study, in order to determine whether tigecycline restricts the selective enrichment of resistant mutant subpopulations of $K$. pneumoniae, we measured the mutant prevention concentrations (MPCs) of tigecycline for $91 \mathrm{~K}$. pneumoniae clinical isolates with four different resistance statuses, and assessed their propensity of developing resistance to tigecycline.

\section{The study}

Ninety one K. pneumoniae isolates were collected from Beijing Hospital, China. The isolates were divided into four groups according to their resistance statuses: (1) thirty-two isolates were carbapenemresistant (resistant to either meropenem, impenem or ertapenem) and fluoroquinolone-resistant (resistant to either ciprofloxacin or levofloxacin); (2) twenty-nine isolates were carbapenem- and fluoroquinolonesusceptible; (3) twenty-two isolates were carbapenemsusceptible and fluoroquinolone-resistant; (4) eight isolates were carbapenem-resistant and fluoroquinolone-susceptible. Staphylococcus aureus ATCC 29213, Escherichia coli ATCC 25922 and K. pneumoniae ATCC BAA-2146 were used as the quality control strain and were included in each susceptibility test.

The minimum inhibition concentrations (MICs) were determined by the method of agar plate assays according to the Clinical and Laboratory Standards Institute guidelines [4]. The MICs for the strains were interpreted in accordance with FDA guidelines for tigecycline, MIC $\leq 2 \mu \mathrm{g} / \mathrm{mL}$ and $\geq 8 \mu \mathrm{g} / \mathrm{mL}$ were classified as susceptible and resistant, respectively [5,6]. MPCs for $K$. pneumoniae of the four groups were performed with a previously described procedure with modifications [7]. Briefly, each isolate was cultured on the Mueller-Hinton (MH) agar and incubated at $37^{\circ} \mathrm{C}$ for 24 hours, then transferred to 500 $\mathrm{mL}$ of $\mathrm{MH}$ and incubated for another 24 hours. The suspension was centrifuged at $5000 \mathrm{~g}$ for 10 minutes. Bacteria were re-suspended with $3 \mathrm{~mL}$ fresh MH broth to a concentration about $10^{10}$ c.f.u. $\mathrm{ml}^{-1}$. Aliquots of $0.1 \mathrm{~mL}$ of the suspension were plated respectively onto a series of agar plates containing various concentrations of tigecycline. After incubation at $37^{\circ} \mathrm{C}$ for 72 hours, the bacterial colonies were counted. The MPC was defined as the lowest drug concentration at which the growth of $K$. pneumoniae on agar plate was completely inhibited. Correlations between MICs and MPCs were analysed using SPSS software, version 18.0 (IBM Corp., Armonk,NY,USA). 
Table 1 shows the distribution of MICs and MPCs of tigecycline for the $91 \mathrm{~K}$. pneumoniae clinical isolates. Using the resistant breakpoint of tigecycline $(8 \mu \mathrm{g} / \mathrm{mL}), 13$ tigecycline-resistant $K$. pneumoniae isolates were obtained, and the tigecycline-resistance rate was $14.3 \%(13 / 91)$. $\mathrm{MPC}_{90}$ and MPC range for the four groups' strains are shown in table 2. The MPCs of tigecycline with $91 \mathrm{~K}$. pneumoniae isolates were 2- to 256-fold higher than the susceptibility breakpoint (2 $\mu \mathrm{g} / \mathrm{mL})$. Furthermore, the MPCs of tigecycline for the cabapenem- and fluoroquinolone-resistant isolates were found to be 8-fold higher than those for cabapenem and quinolones-susceptible isolates. However, there was no significant difference of the MPC values between cabapenem-resistant and quinolones-susceptible and cabapenem-susceptible and quinolones-resistant isolates. As shown in table 1, the MPCs for the isolates ranged over 6 2-fold dilutions even with the same MIC values. In addition, low correlations between MPCs and MICs were observed for all the91 K. pneumoniae isolates $\left(\mathrm{r}^{2}=\right.$ $0.53)$, or the 13 tigecycline-resistant isolates $\left(r^{2}=\right.$ 0.32 ), as well as the 78 tigecycline-susceptible isolates $\left(r^{2}=0.29\right)$, which is consistent with previous reports. [8]

Conte JE et al.[9] reportedthat the maximum concentration of drug in serum $\left(\mathrm{C}_{\max }\right)$ and in alveolar cells was $0.38 \pm 0.06 \mu \mathrm{g} \mathrm{ml}-1$ and $15.2 \pm 7.6 \mu \mathrm{g} \mathrm{ml}-1$, respectively, after intravenous injection administration of the recommended dosage of $50 \mathrm{mg}$ tigecycline every 12 hours. Our data showed that $\mathrm{MPC}_{90}$ values of tigecycline were $128 \mathrm{mg} \mathrm{ml}^{-1}$ and $32 \mathrm{mg} \mathrm{ml}^{-1}$ for cabapenem and quinolones-resistant and cabapenem and quinolones-susceptible $K$. pneumoniae clinical isolates,respectively, which were much higher than the tigecycline concentrations in serum and lung tissue.

Table 1. Distribution of MICs and MPCs of tigecycline for the K. pneumoniae clinical isolates.

\begin{tabular}{|c|c|c|c|c|c|c|c|c|c|c|}
\hline \multirow{2}{*}{$\begin{array}{l}\text { Minimum inhibitory } \\
\text { concentrations (MICs), } \mu \mathrm{g} / \mathrm{ml}\end{array}$} & \multicolumn{10}{|c|}{ Mutant prevention concentrations (MPCs), $\mu \mathrm{g} / \mathrm{ml}$} \\
\hline & $0.25-2$ & 4 & 8 & 16 & 32 & 64 & 128 & 256 & 512 & $\mathbf{N}$ \\
\hline 0.25 & & & & 1 & & & & & & 1 \\
\hline 0.5 & & 2 & 10 & 3 & 2 & & & & & 17 \\
\hline 1 & & 3 & 9 & 15 & 5 & & & & & 32 \\
\hline 2 & & 2 & 3 & 8 & 3 & 1 & 1 & & & 18 \\
\hline 4 & & & & 3 & 2 & 4 & 1 & & & 10 \\
\hline 8 & & & & 1 & 1 & 1 & 2 & & & 5 \\
\hline 16 & & & & & 1 & 2 & & & & 3 \\
\hline 32 & & & & 1 & & & 1 & 2 & 1 & 5 \\
\hline $\mathrm{N}$ & & 7 & 22 & 32 & 14 & 8 & 5 & 2 & 1 & 91 \\
\hline
\end{tabular}

Table 2. Comparison of the MICs before and after mutant prevention concentration and MPCs of K. pneumoniae isolates with four different resistance statuses.

\begin{tabular}{|c|c|c|c|c|c|c|c|c|}
\hline \multirow{2}{*}{$\begin{array}{c}\text { Antimicrobial } \\
\text { Background (N) }\end{array}$} & \multicolumn{3}{|c|}{ Before mutant prevention concentration } & \multicolumn{3}{|c|}{ After mutant prevention concentration } & \multirow[b]{2}{*}{ MPC range } & \multirow[b]{2}{*}{ MPC $_{9}$} \\
\hline & MIC range & $\mathrm{MIC}_{50}$ & $\mathrm{MIC}_{90}$ & MIC range & $\mathrm{MIC}_{50}$ & $\overline{\text { MIC }_{90}}$ & & \\
\hline All $(\mathrm{N}=91)$ & $0.25-32$ & 1 & 8 & $2-128$ & 8 & 16 & $4-512$ & 64 \\
\hline TGC-R (N=13) & $8-32$ & 16 & 32 & $4-128$ & 16 & 128 & $16-512$ & 256 \\
\hline TGC-S (N=78) & $0.25-4$ & 1 & 4 & $2-32$ & 2 & 16 & $4-128$ & 32 \\
\hline Carbapenems (R) & & & & & & & & \\
\hline $\begin{array}{c}\text { Quinolones }(\mathrm{R}) \\
\quad(\mathrm{N}=32)\end{array}$ & $0.5-32$ & 1 & 32 & $2-128$ & 8 & 16 & $4-512$ & 128 \\
\hline $\begin{array}{c}\text { Carbapenems (S) } \\
\text { Quinolones (S) } \\
(\mathrm{N}=29)\end{array}$ & $0.25-8$ & 1 & 4 & $2-32$ & 8 & 16 & 4-64 & 32 \\
\hline $\begin{array}{c}\text { Carbapenems (R) } \\
\text { Quinolones(S) } \\
(\mathrm{N}=8)\end{array}$ & $0.5-4$ & 1 & 16 & $2-16$ & 8 & 16 & $8-128$ & 128 \\
\hline $\begin{array}{c}\text { Carbapenems (S) } \\
\text { Quinolones (R) } \\
(\mathrm{N}=22)\end{array}$ & $0.5-16$ & 1 & 16 & $2-32$ & 16 & 16 & $4-128$ & 64 \\
\hline
\end{tabular}

TGC-R: tigecycline-resistance; TGC-S: tigecycline-susceptible; MIC: minimum inhibitory concentration; MPC: mutant prevention concentration; MIC $_{50}$ : Drug concentration inhibiting $50 \%$ of the isolates tested; $\mathrm{MIC}_{90}$ and $\mathrm{MPC}_{90}$ : Drug concentration inhibiting $90 \%$ of the isolates tested. 
Therefore, at the recommended dosage, the tigecycline concentrations would fall into the mutant selective window (drug concentration range between MIC and MPC), and likely to lead to the enrichment of resistant mutant subpopulations. Our result are in agreement with a recent study from Korea, by Myung-Jin Choi et al.[10]; they reported that the MICs and MPCs of tigecycline for $K$. pneumoniae isolates ranged between $0.5-1 \mathrm{mg} / \mathrm{L}$ and $4-16 \mathrm{mg} / \mathrm{L}$, respectively, suggesting that the current clinical dosage regimen may lead to the development of tigecycline-resistant mutants.

The following limitations of our study should be considered. First, all of the studied isolates were recovered from a single hospital, which might increase the biases of our results. Second, Myung-Jin Choi et al. [10] found that up-regulation of the efflux pumps was associated with tigecycline resistance. The efflux pump expression levels were not tested in the singlestep mutants in our study. Further studies are warranted to validate the relationship between efflux pump expression and tigecycline resistance.

\section{Conclusions}

Our data indicate that tigecycline therapy may be prone to the emergence of resistance with $K$. pneumoniae. Therefore, the continuous monitoring of $K$. pneumoniae susceptibility and patients responsiveness to tigecycline treatment is recommended.

\section{Acknowledgements}

This work was supported by The Significant Creation of New Drugs of the Ministry of National Science \& Technology's the Twelfth Five-Year Plan-Research Project on the Clinical Evaluation Technology Platform of Cardio-cerebrovascular Disease (2012ZX09303-008-002).

\section{References}

1. Petrosillo N, Giannella M, Lewis R, Viale P (2013) Treatment of carbapenem-resistant Klebsiella pneumoniae: the state of the art. Expert Rev Anti Infect Ther 11: 159-177.

2. Sun Y, Cai Y, Liu X, Bai N, Liang B, Wang R (2013) The emergence of clinical resistance to tigecycline. Int $\mathrm{J}$ Antimicrob Agents 41:110-116.

3. Zhong X, Xu H, Chen D, Zhou H, Hu X, Cheng G (2014) First emergence of acr $\mathrm{AB}$ and oqxAB mediated tigecycline resistance in clinical isolates of Klebsiella pneumoniae predating the use of tigecycline in a Chinese hospital. PLoS One 9: e115185.

4. Clinical and Laboratory Standards Institute (2014) Performance Standards for Antimicrobial Susceptibility Testing; Twenty-Fourth Informational Supplement. CLSIdocument M100-S24. Wayne, PA: Clinical and Laboratory Standards Institute, 2014.

5. Anthony KB, Fishman NO, Linkin DR, Gasink LB, Edelstein PH, Lautenbach E (2008) Clinical and microbiological outcomes of serious infections with multidrug-resistant gramnegative organisms treated with tigecycline. Clin Infect Dis 46: 567-570.

6. Schafer JJ, Goff DA, Stevenson KB, Mangino JE (2007) Early experience with tigecycline for ventilator-associated pneumonia and bacteremia caused by multidrug-resistant Acinetobacter baumannii. Pharmacotherapy 27: 980-987.

7. Blondeau JM, Zhao X, Hansen G, Drlica K (2001) Mutant prevention concentrations of fluoroquinolones for clinical isolates of Streptococcus pneumoniae. Antimicrob Agents Chemother 45: 433-438.

8. Drlica K, Zhao X, Blondeau JM, Hesje C ( 2006) Low correlation between mic and mutant prevention concentration. Antimicrob Agents Chemother 50:403-404.

9. Conte JE Jr., Golden JA, Kelly MG, Zurlinden E (2005) Steady-state serum and intrapulmonary pharmacokinetics and pharmacodynamics of tigecycline. Int J Antimicrob Agents 25: 523-529.

10. Choi MJ, Peck KR, Ko KS (2015) Mutant prevention concentration of tigecycline for Acinetobacter baumannii and Klebsiella pneumoniae clinical isolates. J Antimicrob Chemother 70: 621-622.

\section{Corresponding author}

Xin Hu, Department of Pharmacy, Beijing Hospital, No1, Dahua Road, Dongdan Street 100730 Beijing, China

Phone: (8610) 85133636

Email: huxinbjyy@hotmail.com

Conflict of interests: No conflict of interests is declared. 